\title{
DETECTABILITY OF RED-EDGE-SHIFTED VEGETATION ON TERRESTRIAL PLANETS ORBITING M STARS
}

\author{
Giovanna Tinetti \\ European Space Agency/Institut d'Astrophysique de Paris, Extrasolar Planets Section, Paris, France 75014; tinetti@iap.edu \\ AND \\ SKY RASHBY AND YUK L. YUNG \\ California Institute of Technology, Division of Geological and Planetary Sciences, Pasadena, CA 91125; sky@gps.caltech.edu, yly@gps.caltech.edu \\ Received 2006 February 3; accepted 2006 May 12; published 2006 June 13
}

\begin{abstract}
We have explored the detectability of exovegetation on the surface of a terrestrial planet orbiting an $\mathrm{M}$ star. The exovegetation is responsible for producing a pigment-derived surface signature that is redshifted with respect to the Earth vegetation's red edge. The redshift was estimated using a model of leaf optical property spectra (Jacquemoud \& Baret) combined with a 3 photon photosynthetic scheme calculated by Wolstencroft \& Raven for a possible exovegetation growing on an $\mathrm{M}$ star planet. To study the detectability of this surface biosignature on an M star terrestrial planet, we have used the three-dimensional model developed by Tinetti et al. for the case of the Earth. This model can generate disk-averaged spectra and broadband integrated fluxes, which will be useful for future terrestrial planet exploration missions, such as the NASA Terrestrial Planet Finder Coronagraph. Input to this model were the atmospheric profiles and cloud distributions predicted by Joshi and coworkers for a synchronous planet orbiting an $\mathrm{M}$ dwarf and the distinctive surface reflectance of the exovegetation. While on Earth this pigment-derived surface feature would be almost completely masked by water absorption, even in a cloud-free atmosphere, we found that the strength of the edge feature on our simulated $\mathrm{M}$ star terrestrial planet can exceed that on Earth, given the right conditions. Obviously, the detectability of such biosignatures would be highly dependent on the extent of vegetation surface area, cloud cover, and viewing angle.
\end{abstract}

Subject headings: astrobiology — atmospheric effects — radiative transfer — stars: late-type

Online material: color figure

\section{INTRODUCTION}

The search for habitable environments and life beyond the Earth is listed as one of the principal scientific objectives in the NASA and ESA long-term vision for space exploration. To support their vision, NASA and ESA plan to launch a series of missions over the next few decades that will have the capability to detect and characterize terrestrial-mass planets around nearby stars and will search for other habitable environments and life. In particular, the NASA Terrestrial Planet Finder Coronagraph (TPF-C, nominal launch 2014) will use visible (VIS) and nearinfrared (NIR) radiation to search for extrasolar planets down to sub-Earth size and will perform a multiwavelength preliminary characterization of planetary atmospheres and surfaces at relatively low spectral resolution $(R \sim 70)$. Once detected by $T P F$, the extreme distance to the planet precludes us from being able to spatially resolve the planet's disk: the planet's surface and atmospheric composition, its physical properties, and the possible presence of life must all be inferred from a disk-averaged spectrum (Beichman et al. 1999). ${ }^{1}$

On Earth, the red edge is a distinctive signature of vegetation. Technically, the red edge is a spectral reflectance feature characterized by darkness in the red portion of the visible spectrum, due to absorption by chlorophyll, contrasting strongly with high reflectance in the NIR, due to light scattering from refraction along interfaces between leaf cells and air spaces inside the leaf (Gates et al. 1965; Horler et al. 1983). Astrobiologists are interested to know whether or not a similar biosignature could arise on an extrasolar planet and thus be detectable by future space missions (Arnold et al. 2002; Woolf et al. 2002; Schneider 2003; Seager et al. 2005; Montañés-Rodriguez et al. 2005;

\footnotetext{
${ }^{1}$ See http://planetquest.jpl.nasa.gov/TPF/tpf_index.html.
}

Tinetti et al. 2006b; Turnbull et al. 2005). Based on knowledge of photosynthesis on Earth and of stellar evolution, Wolstencroft \& Raven (2002) conclude that it is likely that photosynthesis would have evolved on Earth-like planets in response to the same evolutionary factors as have been involved on Earth. Their model of photosynthesis on Earth-like planets orbiting stars of different spectral types shows that cooler stars, with maximum radiation output at longer wavelengths (Fig. 1), may require more than the 2 photons per electron that are transferred and that are used in oxygen-evolving photosynthesis on Earth. Detection of photosynthesis will be based on spectroscopy using most probably photosynthetic pigments that could have very different absorption properties from those on Earth. For these reasons, we have explored the detectability of an "exovegetation" responsible for producing a red-edge-like signature that is redshifted with respect to that of Earth's vegetation. The redshift was estimated using a model of leaf optical property spectra (Jacquemoud \& Baret 1990) combined with the 3 photon photosynthetic scheme calculated by Wolstencroft \& Raven (2002).

To study the detectability of this surface biosignature both in the case of Earth and, more appropriately, on an M star terrestrial planet with plausible atmospheric properties, we have used the three-dimensional model developed by Tinetti et al. (2005, 2006a), for the case of Mars and the Earth. This model can generate disk-averaged spectra and broadband integrated fluxes, which will be useful for future terrestrial planet exploration missions, such as TPF-C. To simulate the atmospheric profiles and cloud distributions of a synchronous terrestrial planet orbiting an M star, we have used the predictions of Joshi et al. (1997) and Joshi (2003). M star systems are not easy to study with $T P F$ missions, since their habitable zone lies very close to their parent stars, requiring high spatial resolution to separate star from 


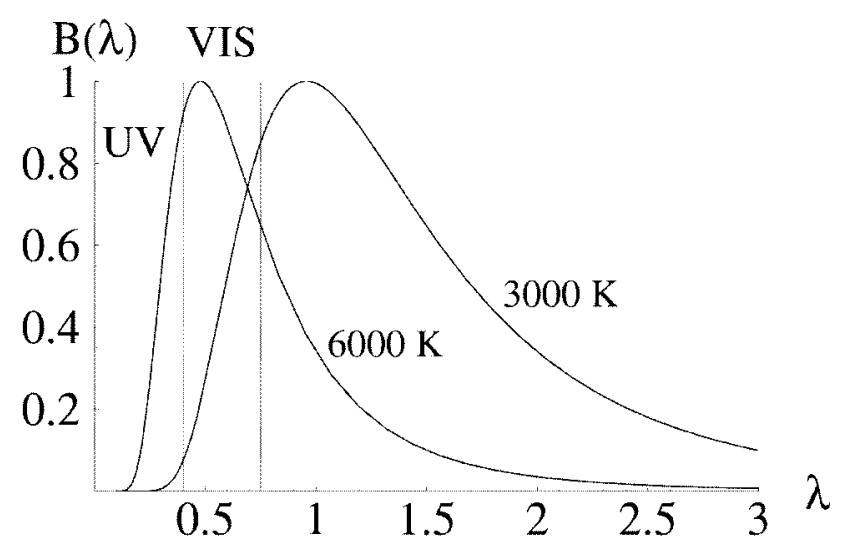

FIG. 1.-Blackbody radiances at 3000 and $6000 \mathrm{~K}$ computed using formula (2.38) of Goody \& Yung (1989), normalized to the maxima. Vertical lines indicate the visible range $(0.4-0.7 \mu \mathrm{m})$.

planet. However, $T P F-\mathrm{C}$ might be able to observe at least part of the habitable zone around a few M stars. ${ }^{2}$

\section{MODEL}

Exovegetation utilizing the proposed 3 photon biochemistry (Wolstencroft \& Raven 2002) was simulated by modifying the plate model of leaf optical properties, Prospect, developed by Jacquemoud \& Baret (1990). Specifically, photopigments were assumed to absorb strongly below $1050 \mathrm{~nm}$ and to rapidly attenuate in absorption beyond this point. The use of a third photon, in fact, allows the oxidation of water to be coupled to the reduction of an electron carrier with a potential similar to that of NADP + , which could subsequently be used to fix carbon and accumulate biomass. Assuming a quantum yield similar to that of terrestrial vegetation, Wolstencroft \& Raven (2002) calculate a maximum wavelength of $1050 \mathrm{~nm}$ for each of the 3 photons utilized in such a system.

The use of a leaf model implies an exovegetation that has complex leaflike structures almost exactly like the laminate leaves of higher plants on Earth. Convergent evolution has apparently favored the development of these structures (Boyce

${ }^{2}$ A list of possible TPF target stars may be found at http://planetquest .jpl.nasa.gov/Navigator/library/basdtp.pdf.

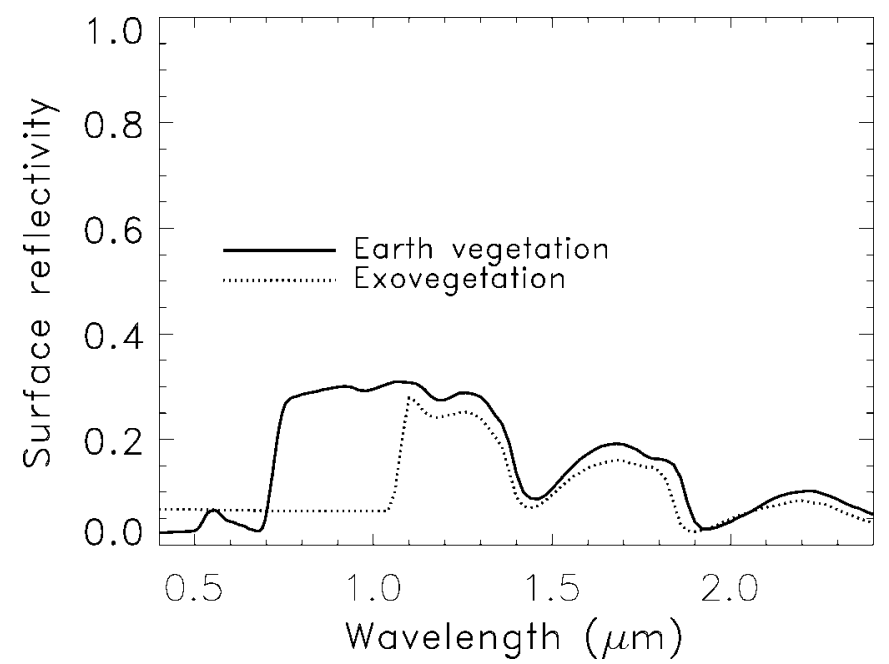

FIG. 2.-Redshifted edge (dotted line), as modeled with Prospect, compared with the Earth's vegetation red edge (solid line).

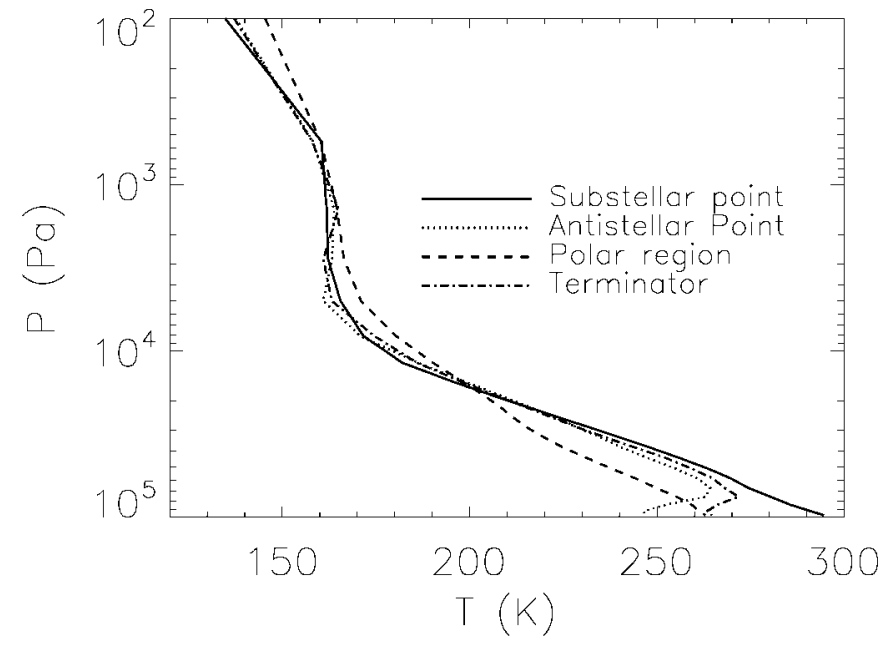

FIG. 3.-Examples of temperature-pressure profiles calculated by Joshi et al. (1997) using a general circulation model.

\& Knoll 2002), consistent with our understanding of leaves as an extremely efficient means of balancing energy generation and heat dissipation. The development of multicellular lightharvesting structures is likely to have considerable ramifications in terms of geochemical feedbacks and planetary oxygenation time (Beerling 2005; Catling et al. 2005) and would significantly improve the odds of remote detection by amplifying edge features associated with photosynthetic pigments.

In our calculations, standard values based on terrestrial vegetation were assumed for leaf dry matter, water content, and structural characteristics. These parameters may affect, though, only the vertical variations in the spectral reflectances, hence the strength of the signal. The wavelength at which the edge occurs is determined by the photopigment absorption properties (Kiang et al. 2006). The result (see Fig. 2) was a whole-leaf spectrum that lacks the edge feature of normal terrestrial vegetation just beyond $700 \mathrm{~nm}$ (solid line) but that possesses a similar feature at $1050 \mathrm{~nm}$. The effect of leaf structure in both cases is to amplify the effect of selective pigment absorption.

To simulate the disk-averaged spectra of the Earth where all vegetation is replaced with the exovegetation, we used the mod-

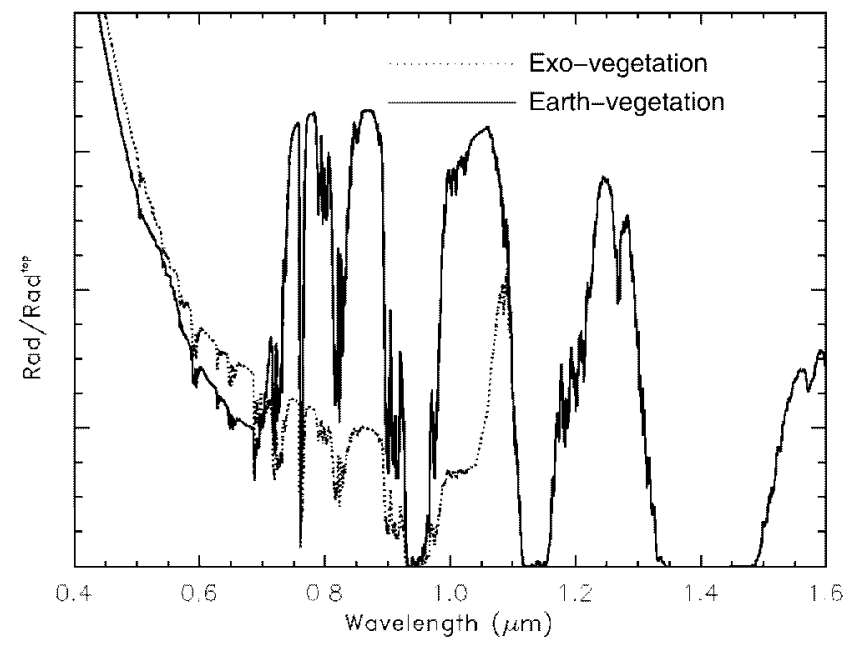

FIG. 4.-Comparison of normal and shifted red-edge signatures in the VISNIR cloud-free spectra of the Earth. The redshifted one shows a weaker signal, partially obscured by water absorption bands. 

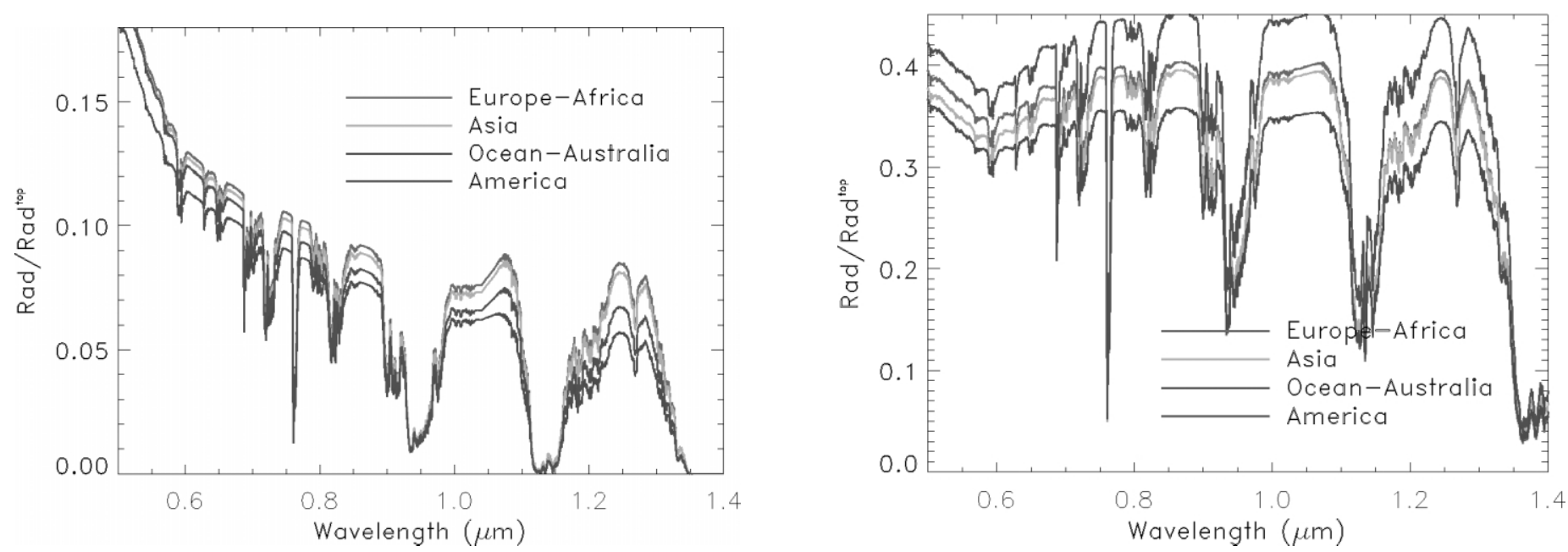

FIG. 5.-Disk-averaged spectra in the VIS and NIR of a fully illuminated cloud-free Earth (left) and of a cloudy Earth (right) seen from different views following the diurnal rotation of the planet. By changing the viewing geometries that are visible during the diurnal rotation, the land vs. ocean fraction changes considerably with time, hence the redshifted red-edge detectability in the band $\geq 1 \mu \mathrm{m}$. Clouds may obscure this signature (Tinetti et al. 2006b). [See the electronic edition of the Journal for a color version of this figure.]

els and Earth atmospheric and surface data described in Tinetti et al. (2006a). In detail, we first compiled a spatially resolved, global description of the Earth's surface and atmospheric properties that affect its spectrum. These properties include surface albedos, atmospheric trace gases, clouds, and temperature distributions. Here this information was compiled from available observations and modeling results (Fishbein et al. 2003). These properties were then mapped onto the globe at high spatial resolution and then subsampled using the Hierarchical Equal Area and isoLatitude Pixelization (HEALPix) approach (Górski et al. 1999). ${ }^{3}$ Spectrally resolved radiances were then derived at each of these spatial grid points, using the Spectral Mapping Atmospheric Radiative Transfer (SMART) model (Meadows \& Crisp 1996; Crisp 1997). Finally, by specifying the spatial resolution and observing geometry, a final program selected

${ }^{3}$ See http://healpix.jpl.nasa.gov/.

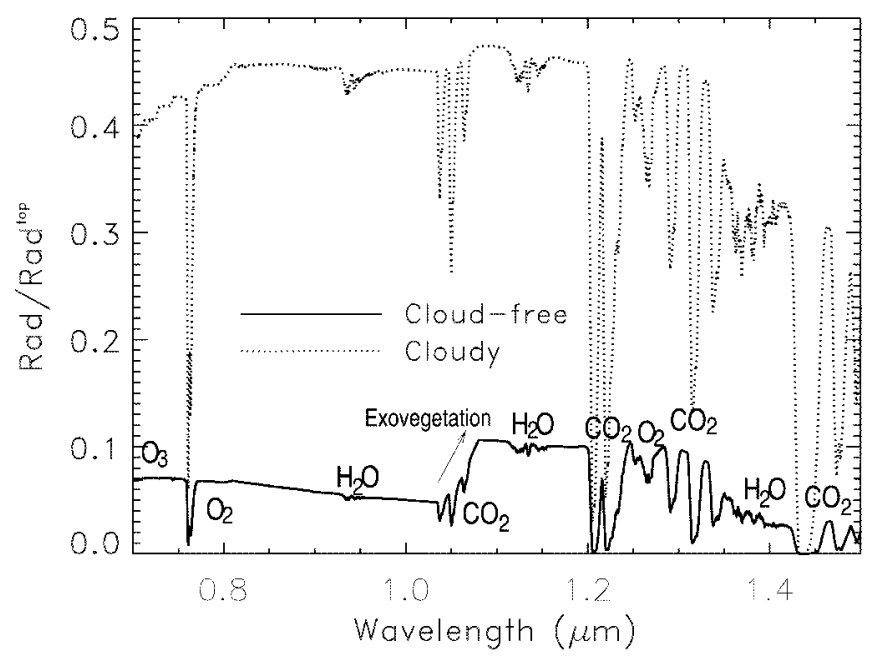

FIG. 6.-Disk-averaged spectrum in the VIS and NIR of an hypothetical terrestrial planet orbiting a class $\mathrm{M}$ star as described by Joshi et al. In the absence of clouds, the $1050 \mathrm{~nm}$ surface signature is again visible (solid line). The addition of clouds decreases the strength of the signature much like the Earth case, despite markedly different cloud types (dotted line). the appropriate synthetic spectra, mapped them to the disk, and then integrated the results to yield a disk-averaged spectrum.

By contrast, the terrestrial planet orbiting the $\mathrm{M}$ dwarf is assumed to be in close proximity to its parent star, and thus tidally locked, and the mixing ratio of $\mathrm{CO}_{2}$ in the atmosphere is increased to 0.8 bars at the expense of $\mathrm{N}_{2}$ to prevent atmospheric condensation. The temperature and pressure profiles and cloud distributions were calculated by Joshi et al. (1997) and Joshi (2003) using a general circulation model (Fig. 3). We chose the data relative to the illuminated portion of the planet since it is the only area that is likely to be covered by exovegetation. In this area, according to Joshi's calculations, low clouds cover $22 \%$ of the surface, middle clouds $25 \%$, high clouds $25 \%$, and convective clouds $47 \%$. Convective clouds only form parcels above $231 \mathrm{~K}$, and at pressures above 200 mbars, and they may overlap other cloud types. The M star spectrum used for the radiative calculations was taken from Leggett et al. (2000), and it corresponds to an effective surface temperature of $\sim 3100 \mathrm{~K}$.

\section{DETECTABILITY OF VEGETATION SIGNATURE}

Earth disk-averaged spectra, where all vegetation was replaced with exovegetation (Fig. 4), were simulated from multiple viewing angles (Fig. 5). In the Earth case, the primary challenges to detecting the redshifted edge are the spatial extent of exovegetation over the planetary surface and the question of how much it might be obscured by clouds and water. The edge feature is visible (as an increase in outgoing/incoming radiation beyond $\sim 1 \mu \mathrm{m}$ ) when clouds are excluded from the model (Fig. 5, left panel). The effect of varied surface coverage is observed in the difference between viewing angles dominated by continents or ocean. However, the redshifted edge signature is peaking in a band where water absorption occurs, masking part of the signal. The result is a weak increase of the albedo in a narrow band without a distinctive/unique signature. The inclusion of realistic clouds (Fig. 5, right panel) significantly weakens the signature at $\sim 1 \mu \mathrm{m}$.

We also investigated the detectability of the exovegetation on a terrestrial planet orbiting an M star (see $\S 2$ ). The planet, being tidally locked, is assumed to be entirely covered by exovegetation on the illuminated portion of the surface. In the 
cloud-free case (Fig. 6, solid line), the shifted edge causes a rise in the signal of $70 \%$, due to the fact that the atmospheric conditions show less water absorption. However, when clouds are accounted for in a realistic way (Fig. 6, dotted line), the signal is increased by $\sim 10 \%$ only. The signal might be even weaker if the exovegetation were sparsely distributed on the surface that is exposed to the central star, if the leaf structure were different, or if the phase observed is not the fully illuminated one but rather the gibbous one or the dichotomy.

\section{CONCLUSIONS}

We have demonstrated that, under the right conditions, a photopigment-derived surface biosignature similar to the terrestrial red edge should be detectable beyond our solar system, if it exists. The detectability of such a biosignature would be highly dependent on the nature and extent of cloud cover as well as viewing angle and vegetation surface area. Nonetheless, the strength of the edge feature on extrasolar planets could potentially exceed that on Earth in some cases, and the development of more comprehensive models and complementary techniques may be significantly beneficial to future astrobiological missions.

We would like to thank M. Joshi, V. S. Meadows, D. Crisp, and R. L. Shia for their valuable inputs, X. Jiang, V. Natraj, M. C. Liang, X. Guo, M. Gerstell, and C. D. Parkinson for useful comments, and the referee for improvements to the Letter. Part of this work was performed at the California Institute of Technology. G. Tinetti is currently sponsored by the European Space Agency. Y. L. Yung was partly supported by the National Aeronautics and Space Administration through the NASA Astrobiology Institute (CAN-00-OSS-01) and a NASA grant (NASA 5-13296).

\section{REFERENCES}

Arnold, L., Gillet, S., Lardière, O., Riaud, P., \& Schneider, J. 2002, A\&A, 392, 231

Beerling, D. J. 2005, Ann. Botany, 96(3), 345

Beichman, C. A., Woolf, N. J., \& Lindensmith, C. A., eds. 1999, The Terrestrial Planet Finder (TPF) (JPL Publ. 99-3; Pasadena: JPL)

Boyce, C. K., \& Knoll, A. H. 2002, Paleobiology, 28(1), 70

Catling, D. C., Glein C. R., Zahnle, K. J., \& McKay, C. P. 2005, Astrobiology, 5(3), 415

Crisp, D. 1997, Geophys. Res. Lett., 24, 571

Fishbein, E., et al. 2003, IEEE Trans. Geosci. Remote Sensing, 41(2), 314

Gates, D. M., Gates, H. J., Gates, J. C., \& Gates, V. R. 1965, Appl. Opt., 4(1), 11

Goody, R. M., \& Yung, Y. L. 1989, Atmospheric Radiation: Theoretical Basis (New York: Oxford Univ. Press)

Górski, K. M., Hivon, E., \& Wandelt, B. D. 1999, in Evolution of Large Scale Structure: From Recombination to Garching, ed. A. J. Banday, R. K. Sheth, \& L. Da Costa (Ipskamp: Printpartners), 37

Horler, D. N. H., Dockray, M., Barber, J., \& Barringer, A. R. 1983, Adv. Space Res., 3(2), 273

Jacquemoud, S., \& Baret, F. 1990, Remote Sensing Environ., 34, 75

Joshi, M. 2003, Astrobiology, 3(2), 415

Joshi, M. M., Haberle, R. M., \& Reynolds, R. T. 1997, Icarus, 129, 450
Kiang, N., Segura, A., Siefert, J., Tinetti, G., Cohen, M., \& Meadows, V. S. 2006, Astrobiology, submitted

Leggett, S. K., Allard, F., Dahn, C., Hauschildt, P. H., Kerr, T. H., \& Rayner, J. 2000, ApJ, 535, 965

Meadows, V. S., \& Crisp, D. 1996, J. Geophys. Res., 101, 4595

Montañés-Rodriguez, P., Pallé, E., Goode, P. R., Hickey, J., \& Koonin, S. E. 2005, ApJ, 629, 1175

Schneider, J. 2003, in Towards Other Earths: DARWIN/TPF and the Search for Extrasolar Terrestrial Planets, ed. M. Fridlund \& T. Henning (ESA SP539; Noordwijk: ESA), 205

Seager, S., Turner, E. L., Schafer, J., \& Ford, E. B. 2005, Astrobiology, 5(3), 372

Tinetti, G., Meadows, V. S., Crisp, D., Fong, W., Fishbein, E., Turnbull, M., \& Bibring, J.-P. 2006a, Astrobiology, 6(1), 34

Tinetti, G, Meadows, V. S., Crisp, D., Fong, W., Velusamy, T., \& Snively, H. 2005, Astrobiology, 5(4), 461

Tinetti, G., Meadows, V. S., Crisp, D., Kiang, N., Kahn, B., Bosc, E., \& Velusamy, T. 2006b, Astrobiology, in press

Turnbull, M. C., Traub, W. A., Jucks, K. W., Woolf, N. J., Meyer, M. R., Gorlova, N., Skrutskie, M. F., \& Wilson, J. C. 2005, ApJ, submitted

Wolstencroft, R. D., \& Raven, J. A. 2002, Icarus, 157, 535

Woolf, N. J., Smith, P. S., Traub, W. A., \& Jucks, K. W. 2002, ApJ, 574, 430 